TITLE:

\title{
Perfect Abelian dominance of confinement in quark-antiquark potential in SU(3) lattice QCD
}

\author{
$\operatorname{AUTHOR}(\mathrm{S})$ :
}

Suganuma, Hideo; Sakumichi, Naoyuki

\section{CITATION:}

Suganuma, Hideo ...[et al]. Perfect Abelian dominance of confinement in quark-antiquark potential in SU(3) lattice QCD. AIP Conference Proceedings 2016, 1701(1): 020016.

\section{ISSUE DATE:}

2016-01-22

URL:

http://hdl.handle.net/2433/229492

\section{RIGHT:}

(c) 2016 AIP Publishing. This article may be downloaded for personal use only. Any other use requires prior permission of the author and AIP Publishing. The following article appeared in 'AlP Conference Proceedings 1701, 020016 (2016)' and may be found at http://aip.scitation.org/doi/abs/10.1063/1.4938605; The full-text file will be made open to the public on 22 Jan 2017 in accordance with publisher's 'Terms and Conditions for Self-Archiving'. 


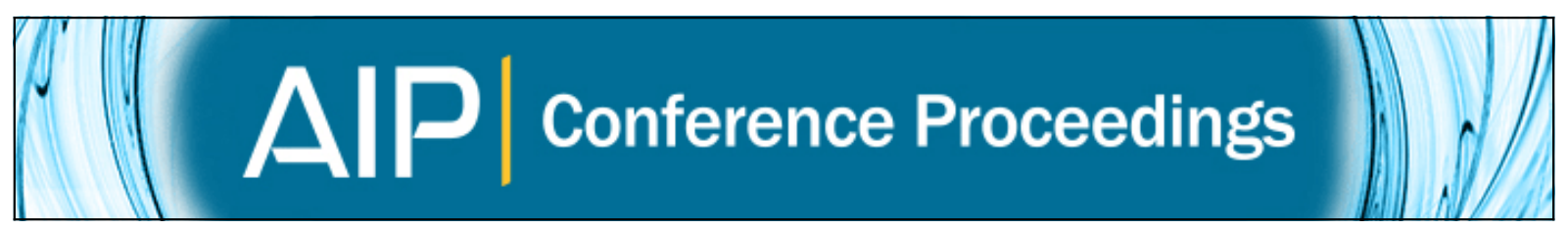

Perfect Abelian dominance of confinement in quark-antiquark potential in SU(3) lattice QCD

Hideo Suganuma and Naoyuki Sakumichi

Citation: AIP Conference Proceedings 1701, 020016 (2016); doi: 10.1063/1.4938605

View online: http://dx.doi.org/10.1063/1.4938605

View Table of Contents: http://scitation.aip.org/content/aip/proceeding/aipcp/1701?ver=pdfcov

Published by the AIP Publishing

\section{Articles you may be interested in}

On quark-antiquark approximation in light front QCD with zero gluon modes

AIP Conf. Proc. 1701, 040023 (2016); 10.1063/1.4938640

Quark-antiquark pairs in the quark model

AIP Conf. Proc. 1361, 171 (2011); 10.1063/1.3622695

SU(3) lattice QCD study for static three-quark potential

AIP Conf. Proc. 594, 341 (2001); 10.1063/1.1425520

The quark-antiquark potential and quantum chromodynamics

AIP Conf. Proc. 74, 370 (1981); 10.1063/1.33104

The spectroscopy of heavy quark-antiquark systems

AIP Conf. Proc. 43, 387 (1978); 10.1063/1.31310 


\title{
Perfect Abelian dominance of confinement in quark-antiquark potential in SU(3) lattice QCD
}

\author{
Hideo Suganuma* and Naoyuki Sakumichi ${ }^{\dagger}$ \\ *Department of Physics, Kyoto University, Kitashirakawaoiwake, Sakyo, Kyoto 606-8502, Japan \\ ${ }^{\top}$ Theoretical Research Division, Nishina Center, RIKEN, Wako, Saitama 351-0198, Japan
}

\begin{abstract}
In the context of the dual superconductor picture for the confinement mechanism, we study maximally Abelian (MA) projection of quark confinement in SU(3) quenched lattice QCD with $32^{4}$ at $\beta=6.4$ (i.e., $a \simeq 0.058 \mathrm{fm}$ ). We investigate the static quark-antiquark potential $V(r)$, its Abelian part $V_{\text {Abel }}(r)$ and its off-diagonal part $V_{\text {off }}(r)$, respectively, from the onaxis lattice data. As a remarkable fact, we find almost perfect Abelian dominance for quark confinement, i.e., $\sigma_{\text {Abel }} \simeq \sigma$ for the string tension, on the fine and large-volume lattice. We find also a nontrivial summation relation of $V(r) \simeq V_{\text {Abel }}(r)+V_{\text {off }}(r)$.
\end{abstract}

Keywords: confinement, lattice QCD, quark potential, dual superconductor

PACS: $12.38 . \mathrm{Aw}, 12.38 . \mathrm{Gc}$

\section{INTRODUCTION}

In 1966, Yoichiro Nambu [1] first proposed the SU(3) gauge theory, i.e., quantum chromodynamics (QCD), as a candidate for the fundamental theory of the strong interaction, just after the introduction of the color quantum number [2]. In 1973, the asymptotic freedom of QCD was theoretically proven [3], and QCD was established as the fundamental theory of the strong interaction via the great success of perturbative QCD to high-energy hadron reactions. Even at present, however, analytical treatment of QCD is quite difficult in the infrared region, because of its strong-coupling nature there. Actually, in spite of its simple form, QCD creates thousands of hadrons and leads to various interesting nonperturbative phenomena such as color confinement [4] and dynamical chiral-symmetry breaking [5]. Since the first application [6] of lattice QCD Monte Carlo simulations in 1979, lattice QCD has been applied as the direct numerical analysis for nonperturbative QCD [7].

Among the nonperturbative properties of QCD, color confinement is one of the most important subjects remaining in elementary particle physics, and is also an extremely difficult mathematical problem. The difficulty is considered to originate from non-Abelian dynamics and nonperturbative features of QCD, which are largely different from QED. However, it is not clear whether quark confinement is peculiar to the non-Abelian nature of QCD or not.

In 1970's, Nambu, 't Hooft, and Mandelstam proposed an interesting idea that quark confinement might be physically interpreted using the dual version of the superconductivity [4]. In the ordinary superconductor, Cooper-pair condensation leads to the Meissner effect, and the magnetic flux is excluded or squeezed like a quasi-one-dimensional tube as the Abrikosov vortex, where the magnetic flux is quantized topologically. On the other hand, from the Regge trajectory of mesons and baryons, quark confining force in hadrons is characterized by a universal physical quantity of the string tension $\sigma \simeq 0.89 \mathrm{GeV} / \mathrm{fm}$ [8], and lattice QCD calculations also indicates one-dimensional squeezing of the color-electric flux in the QCD vacuum [7]. Therefore, the QCD vacuum could be regarded as the dual version of the superconductor based on above similarities on the low-dimensionalization of the quantized flux between charges.

In the dual-superconductor picture for the QCD vacuum, the squeezing of the color-electric flux between quarks is realized by the dual Meissner effect, as the result of condensation of color-magnetic monopoles, which is the dual version of the electric charge as the Cooper pair. However, there are two large gaps between QCD and the dualsuperconductor picture [9].

1. The dual-superconductor picture is based on the Abelian gauge theory subject to the Maxwell-type equations, where electro-magnetic duality is manifest, while QCD is a non-Abelian gauge theory.

2. The dual-superconductor picture requires condensation of color-magnetic monopoles as the key concept, while QCD does not have such a monopole as the elementary degrees of freedom.

On the connection between QCD and the dual-superconductor scenario, 't Hooft proposed the concept of the Abelian 
gauge fixing [10], a partial gauge fixing which only remains Abelian gauge degrees of freedom in QCD. By the Abelian gauge fixing, QCD reduces into an Abelian gauge theory, where off-diagonal gluons behave as charged matter fields similar to $W_{\mu}^{ \pm}$in the Weinberg-Salam model and give color-electric current $j_{\mu}$ in terms of the residual Abelian gauge symmetry. Remarkably in the Abelian gauge, color-magnetic monopoles appear as topological objects corresponding to the nontrivial homotopy group $\Pi_{2}\left(\mathrm{SU}\left(N_{c}\right) / U(1)^{N_{c}-1}\right)=\mathbf{Z}_{\infty}^{N_{c}-1}$ in a similar manner to the GUT monopole [10, 11]. In general, the monopole appears as a topological defect or a singularity in a constrained Abelian gauge manifold embedded in the compact (and at most semi-simple) non-Abelian gauge manifold.

In fact, by the Abelian gauge fixing, QCD reduces into an Abelian gauge theory including both the electric current $j_{\mu}$ and the magnetic current $k_{\mu}$, which is expected to give a theoretical basis of the dual-superconductor scheme for the confinement mechanism [11, 12, 13, 14]. In particular, in the maximally Abelian (MA) gauge [15], which is a special Abelian gauge, the off-diagonal gluon has a large effective mass of about $1 \mathrm{GeV}[16,17]$, and Abelian dominance of quark confinement is approximately observed in lattice QCD $[18,19,20]$. Then, in the MA gauge, QCD becomes an infrared Abelian gauge theory including the magnetic monopole current $k_{\mu}$ [15] together with the electric current $j_{\mu}$. By the Hodge decomposition, the QCD system can be divided into the monopole part $\left(k_{\mu} \neq 0, j_{\mu}=0\right)$ and the photon part $\left(j_{\mu} \neq 0, k_{\mu}=0\right)$. The lattice QCD studies have shown that the monopole part has quark confinement [21], chiral symmetry breaking [22] and instantons [23], while the photon part does not have all of them. (See Fig.1.) Monopole condensation is also suggested by long entangled monopole worldlines [15, 21] and the magnetic screening [24].

Thus, in the MA gauge, the infrared QCD system resembles a dual superconductor, and the color-magnetic monopole seems to carry the essence of nonperturbative QCD. However, such lattice studies were mainly performed in simplified SU(2) color QCD [9, 15, 16, 18, 19, 20, 21, 22, 23, 24], and there are only a few pioneering studies $[25,26,27]$ on the Abelian dominance of quark confinement in SU(3) color QCD in the real world.

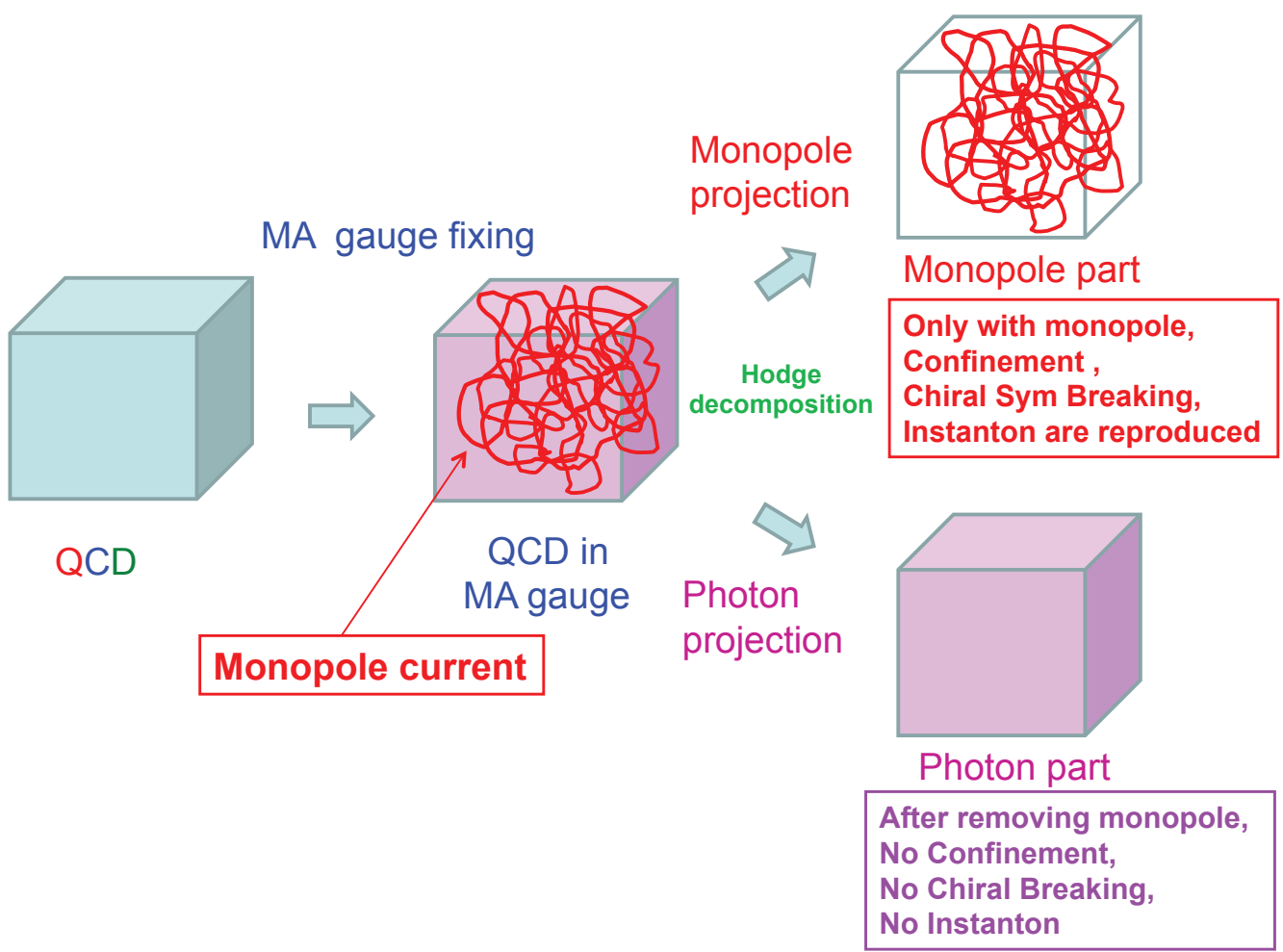

FIGURE 1. A schematic figure of the dual superconductor picture from QCD in the MA gauge. In the MA gauge, QCD becomes Abelian-like because of a large off-diagonal gluon mass of about $1 \mathrm{GeV}$ [16, 17], and monopole currents topologically appear [15]. By the Hodge decomposition, the QCD system in the MA gauge can be divided into the monopole part $\left(k_{\mu} \neq 0, j_{\mu}=0\right)$ and the photon part $\left(j_{\mu} \neq 0, k_{\mu}=0\right)$. The monopole part has quark confinement [21], chiral symmetry breaking [22] and instantons [23], while the photon part does not have all of them, as shown in lattice QCD. 
In this paper, we study Abelian dominance of quark confinement in the MA gauge in SU(3) color QCD at the quenched level on a fine and large-volume lattice of $32^{4}$ at $\beta=6.4$. After the calculation of the quark-antiquark (QQ) potential $V(r)$ and its Abelian part $V_{\text {Abel }}(r)$ in the MA gauge, we consider the fit analysis for them and compare their infrared behavior, and thus investigate quantitatively Abelian dominance of quark confinement in SU(3) QCD.

\section{FORMALISM}

In this section, we briefly review the maximally Abelian (MA) gauge, the Cartan decomposition, and MA projection in $\mathrm{SU}\left(N_{c}\right)$ QCD in both continuum and lattice formalism.

\section{SU(N) continuum QCD}

To begin with, we consider Euclidean continuum QCD and the gluon field $A_{\mu}(x) \in \operatorname{su}\left(N_{c}\right)$. The MA gauge is a special Abelian gauge which minimizes the "global off-diagonal gluon amplitude" [9],

$$
R_{\mathrm{off}}\left[A_{\mu}(\cdot)\right] \equiv \int d^{4} x \operatorname{tr}\left[\hat{D}_{\mu}, \vec{H}\right]\left[\hat{D}^{\mu}, \vec{H}\right]^{\dagger}=\frac{g^{2}}{2} \int d^{4} x \sum_{\alpha}\left|A_{\mu}^{\alpha}(x)\right|^{2}
$$

with the $\mathrm{SU}\left(N_{c}\right)$ covariant derivative $\hat{D}_{\mu} \equiv \hat{\partial}_{\mu}+i g A_{\mu}$ and the Cartan decomposition $A_{\mu}(x)=\vec{A}_{\mu}(x) \cdot \vec{H}+\sum_{\alpha} A_{\mu}^{\alpha}(x) E^{\alpha}$. Thus, in the MA gauge, the off-diagonal gluon amplitude $\left|A_{\mu}^{\alpha}(x)\right|$ is forced to be globally minimized by the gauge transformation, and hence the gluon field $A_{\mu}(x) \equiv A_{\mu}^{a}(x) T^{a}$ maximally approaches the Abelian gauge field $\vec{A} \mu(x) \cdot \vec{H}$.

Since $R_{\text {off }}$ is gauge-transformed by $\Omega \in G$ as

$$
R_{\mathrm{off}} \rightarrow R_{\mathrm{off}}^{\Omega}=\int d^{4} x \operatorname{tr}\left[\Omega \hat{D}_{\mu} \Omega^{\dagger}, \vec{H}\right]\left[\Omega \hat{D}^{\mu} \Omega^{\dagger}, \vec{H}\right]^{\dagger}=\int d^{4} x \operatorname{tr}\left[\hat{D}_{\mu}, \Omega^{\dagger} \vec{H} \Omega\right]\left[\hat{D}^{\mu}, \Omega^{\dagger} \vec{H} \Omega\right]^{\dagger},
$$

the MA gauge fixing condition is obtained as [9]

$$
\left[\vec{H},\left[\hat{D}_{\mu},\left[\hat{D}^{\mu}, \vec{H}\right]\right]\right]=0
$$

from the infinitesimal gauge transformation of $\Omega$.

In the MA gauge, the gauge group $G \equiv \mathrm{SU}\left(N_{c}\right)_{\text {local }}$ is reduced into $\mathrm{U}(1)_{\text {local }}^{N_{c}-1} \times \mathrm{Weyl}_{\text {global }}$, where the global Weyl symmetry is the subgroup of $\mathrm{SU}\left(N_{c}\right)$ relating the permutation of the basis in the fundamental representation [9]. In continuum QCD, MA projection is a simple replacement of the non-Abelian gluon field $A_{\mu}(x) \equiv A_{\mu}^{a}(x) T^{a} \in \operatorname{su}\left(N_{c}\right)$ by its Abelian part $\vec{A}_{\mu}(x) \cdot \vec{H}$.

\section{SU(N) lattice QCD}

Next, let us consider Euclidean lattice QCD formalism, where the gauge field is described by the link-variable $U_{\mu}(s)=e^{i a g A_{\mu}(s)} \in \mathrm{SU}\left(N_{c}\right)$, with the lattice spacing $a$ and the gauge coupling $g$. In lattice QCD, the MA gauge is defined by maximizing the diagonal element of the link-variable,

$$
R_{\mathrm{MA}}\left[U_{\mu}(\cdot)\right] \equiv \sum_{s} \sum_{\mu=1}^{4} \operatorname{tr}\left(U_{\mu}^{\dagger}(s) \vec{H} U_{\mu}(s) \vec{H}\right)
$$

under the $\mathrm{SU}\left(N_{c}\right)$ gauge transformation

$$
U_{\mu}(s) \rightarrow U_{\mu}^{\Omega}(s) \equiv \Omega(s) U_{\mu}(s) \Omega^{\dagger}(s+\hat{\mu}),
$$

with the gauge function $\Omega(s) \in \mathrm{SU}\left(N_{c}\right)$. 
Corresponding to the Cartan decomposition, the $\mathrm{SU}\left(N_{c}\right)$ link-variable $U_{\mu}(s)$ is factorized into a maximal-torussubgroup element $u_{\mu}(s) \in \mathrm{U}(1)^{N_{c}-1}$ and a coset-space element $M_{\mu}(s) \in \mathrm{SU}\left(N_{c}\right) / \mathrm{U}(1)^{N_{c}-1}$ as

$$
U_{\mu}(s)=M_{\mu}(s) u_{\mu}(s) \in G \equiv \operatorname{SU}\left(N_{c}\right),
$$

with

$$
u_{\mu}(s)=e^{i \vec{\theta}_{\mu}(s) \cdot \vec{H}} \in H \equiv \mathrm{U}(1)^{N_{c}-1}, \quad M_{\mu}(s)=e^{i \Sigma_{\alpha} \theta_{\mu}^{\alpha}(s) E^{\alpha}} \in G / H .
$$

In the MA gauge, there remains the residual $\mathrm{U}(1)^{N_{c}-1}$ gauge symmetry, because $R_{\mathrm{MA}}$ in Eq. (4) is invariant under the $\mathrm{U}(1)^{N_{c}-1}$ gauge transformation

$$
U_{\mu}(s) \rightarrow U_{\mu}^{\omega}(s) \equiv \omega(s) U_{\mu}(s) \omega^{\dagger}(s+\hat{\mu})
$$

with $\omega(s) \in \mathrm{U}(1)^{N_{c}-1}$. By the residual gauge transformation (8), $u_{\mu}(s)$ and $M_{\mu}(s)$ transform as

$$
\begin{aligned}
& u_{\mu}(s) \rightarrow u_{\mu}^{\omega}(s) \equiv \omega(s) u_{\mu}(s) \omega^{\dagger}(s+\hat{\mu}), \\
& M_{\mu}(s) \rightarrow M_{\mu}^{\omega}(s) \equiv \omega(s) M_{\mu}(s) \omega^{\dagger}(s),
\end{aligned}
$$

where $M_{\mu}(s)$ keeps the form of $e^{i \sum_{\alpha} \theta_{\mu}^{\alpha}(s) E^{\alpha}} \in G / H$. Thus, the Abelian link-variable $u_{\mu}(s) \in H=\mathrm{U}(1)^{N_{c}-1}$ behaves as the gauge field in $\mathrm{U}(1)^{N_{c}-1}$ lattice gauge theory, which is similar to the compact QED, while the off-diagonal factor $M_{\mu}(s) \in G / H$ behaves as a charged matter field in terms of the residual Abelian gauge symmetry $\mathrm{U}(1)_{\text {local }}^{N_{c}-1}$.

In the lattice QCD, MA projection is defined by the replacement of

$$
U_{\mu}(s) \in G \quad \rightarrow \quad u_{\mu}(s) \in H
$$

\section{LATTICE SETUP AND POTENTIAL MEASUREMENT}

Using the standard plaquette action, we perform SU(3) quenched lattice QCD Monte Carlo calculations on a fine and large-volume lattice of $32^{4}$ at $\beta \equiv 6 / g^{2}=6.4$, i.e., $a \simeq 0.058(7)$ fm. Here, the lattice spacing $a$ is determined so as to reproduce the string tension $\sigma=0.89 \mathrm{GeV} / \mathrm{fm}$ in the inter-quark potential. For comparison, we also investigate a smaller and coarser lattice of $16^{3} \times 32$ at $\beta=6.0$, i.e., $a \simeq 0.105(14) \mathrm{fm}$. After a thermalization of 20000 sweeps, we sample the gauge configuration every 500 sweep, and generate 70 and 250 gauge configurations for $\beta=6.4$ and 6.0 , respectively. On the error estimate, we use the jackknife method.

The (ground-state) quark-antiquark (QQ) potential $V(r)$ at the inter-quark distance $r$ is calculated as

$$
V(r)=-\lim _{T \rightarrow \infty} \frac{1}{T} \ln \left\langle W_{C}\left[U_{\mu}(s)\right]\right\rangle
$$

from the Wilson loop

$$
W_{C}\left[U_{\mu}(s)\right] \equiv \operatorname{tr}\left\{\prod_{C} U_{\mu}(s)\right\}
$$

Here, $C$ denotes the $r \times T$ rectangular loop, and $\langle\cdots\rangle$ means the statistical average over the gauge configurations. For the accurate calculation of the QQ potential, we adopt the gauge-invariant smearing method, which reduces the excited-state components and enhances the ground-state overlap in the QQ system [28, 29].

The Abelian part $V_{\text {Abel }}(r)$, i.e., MA projection of the QQ potential, is obtained as

$$
V_{\text {Abel }}(r)=-\lim _{T \rightarrow \infty} \frac{1}{T} \ln \left\langle W_{C}\left[u_{\mu}(s)\right]\right\rangle
$$

from the Abelian Wilson loop $W_{C}\left[u_{\mu}(s)\right]$ in the MA gauge, which is invariant under the residual Abelian gauge transformation (9) and the global Weyl transformation [9].

Also, we define the off-diagonal part $V_{\text {off }}(r)$ of the QQ potential by

$$
V_{\text {off }}(r)=-\lim _{T \rightarrow \infty} \frac{1}{T} \ln \left\langle W_{C}\left[M_{\mu}(s)\right]\right\rangle
$$


from the off-diagonal Wilson loop $W_{C}\left[M_{\mu}(s)\right]$ in the MA gauge with the $\mathrm{U}(1)^{N_{c}-1}$ Landau gauge, which maximizes

$$
R_{L}\left[u_{\mu}(\cdot)\right] \equiv \sum_{s} \sum_{\mu=1}^{4} \operatorname{Re} \operatorname{tr}\left(u_{\mu}(s)\right)
$$

under the residual gauge transformation (9). (For the argument of perfect Abelian dominance, $V_{\text {off }}(r)$ is not needed.)

For the QQ potential, we here investigate the on-axis data only, and consider the inter-quark distance $2 \leq r \leq 17$ for $\beta=6.4$, and $1 \leq r \leq 8$ for $\beta=6.0$ in the lattice unit. (In Ref.[30], we investigate both on-axis and off-axis data from several different lattices with much more gauge configurations, and find that the main conclusion is almost the same.)

\section{SU(3) LATTICE QCD RESULTS}

We show in Fig.2(a) the SU(3) lattice QCD result of the QQ potential $V(r)$, the Abelian part $V_{\text {Abel }}(r)$ in the MA gauge, and the off-diagonal part $V_{\text {off }}(r)$. The main panels of Fig.2 show the on-axis (integer $r$ ) data on the fine and large-volume lattice of $32^{4}$ at $\beta=6.4$, and the insets those on a smaller and coarser lattice of $16^{3} \times 32$ at $\beta=6.0$.

(a) Cartan decomposition of $\mathrm{Q} \overline{\mathrm{Q}}$ potensial

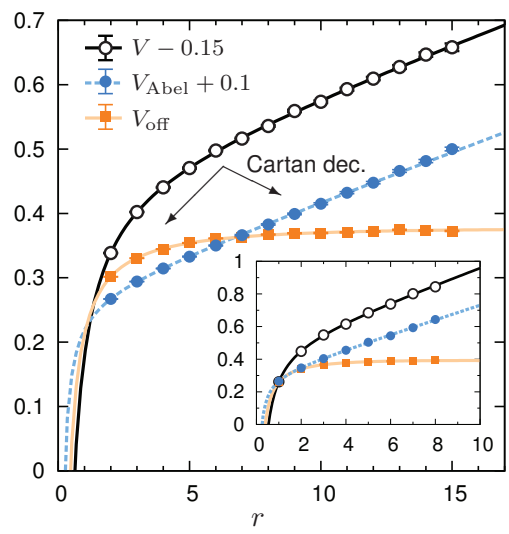

(b) summation formula: $V \simeq V_{\text {Abel }}+V_{\text {off }}$

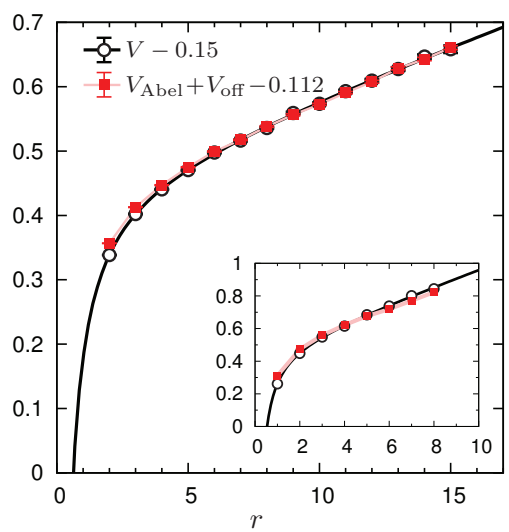

(c) perfect Abelian dominance

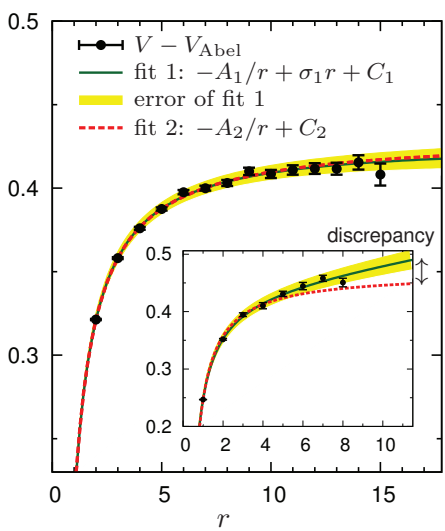

FIGURE 2. (a) The SU(3) lattice QCD result of the QQ potential $V(r)$ (open circles), the Abelian part $V_{\text {Abel }}(r)$ (filled circles) in the MA gauge, and the off-diagonal part $V_{\text {off }}(r)$ (squares). For each part, the best-fit curve with Eq. (17) is added. (b) Comparison between $V_{\text {Abel }}(r)+V_{\text {off }}(r)$ (squares) and $V(r)$ (open circles), except for an irrelevant constant. Their agreement indicates a summation formula of Eq. (18). (c) Fit analysis of $V(r)-V_{\text {Abel }}(r)$ (filled circles) to demonstrate perfect Abelian dominance of quark confinement. The solid curve denotes the best fit with the Coulomb-plus-linear Ansatz of Eq. (17). The dotted curve denotes the best fit with the pure Coulomb Ansatz. For (a), (b), and (c), the main panels show the on-axis (integer $r$ ) data on the fine and large-volume lattice of $32^{4}$ at $\beta=6.4$, and the insets those on a smaller and coarser lattice of $16^{3} \times 32$ at $\beta=6.0$.

\section{Fit analysis with Coulomb-plus-linear Ansatz}

It is known from lattice QCD studies [7, 28, 29] that the QQ $\bar{Q}$ potential $V(r)$ is well reproduced by a sum of the Coulomb and the linear confinement terms,

$$
V(r)=-\frac{A}{r}+\sigma r+C
$$

with the string tension $\sigma$, the color-Coulomb coefficient $A$, and an irrelevant constant $C$.

Then, we carry out the fit analysis for $V(r), V_{\text {Abel }}(r)$, and $V_{\text {off }}(r)$, respectively, based on the Coulomb-plus-linear Ansatz of Eq. (17). We summarize the best-fit parameter set $(\sigma, A, C)$ with $\chi^{2} / N_{\mathrm{df}}$ for the main lattice $\left(32^{4}, \beta=6.4\right)$ and $\left(16^{3} \times 32, \beta=6.0\right)$ in Tables 1 and 2, respectively. In Fig.2(a), we have added the best-fit curves with Eq. (17). 
From the fit analysis, we find for both lattices that the Abelian part $V_{\text {Abel }}(r)$, i.e., MA-projected QQ $\bar{Q}$ potential, can be well fitted with the Coulomb-plus-linear Ansatz, as well as $V(r)$. We also find that the off-diagonal part $V_{\text {off }}(r)$ has almost zero string tension, i.e., $\sigma_{\text {off }} \simeq 0$, and is approximated as a pure Coulomb potential [30].

TABLE 1. Fit analysis with the Coulomb-plus-linear Ansatz for on-axis data of the QQ potentials, $V(r), V_{\text {Abel }}(r)$, and $V_{\text {off }}(r)$, on a fine and large-volume lattice of $32^{4}$ at $\beta=6.4$, i.e., $a \simeq 0.058(7) \mathrm{fm}$. For each potential, the best-fit parameter set $(\sigma, A, C)$ in the functional form of Eq. (17) and $\chi^{2} / N_{\mathrm{df}}$ are listed.

\begin{tabular}{crccc}
\hline & \multicolumn{1}{c}{$\sigma\left[a^{-2}\right]$} & $A$ & $C\left[a^{-1}\right]$ & $\chi^{2} / N_{\mathrm{df}}$ \\
\hline$V$ & $0.01507(20)$ & $0.290(3)$ & $0.603(2)$ & 1.14 \\
$V_{\text {Abel }}$ & $0.01528(14)$ & $0.067(2)$ & $0.170(1)$ & 1.17 \\
$V_{\text {off }}$ & $-0.00038(11)$ & $0.179(3)$ & $0.392(1)$ & 2.41 \\
$V_{\text {Abel }}+V_{\text {off }}$ & $0.01510(15)$ & $0.242(3)$ & $0.560(2)$ & 0.94 \\
\hline
\end{tabular}

TABLE 2. Fit analysis with the Coulomb-plus-linear Ansatz for on-axis data of the QQ potentials $\left(V, V_{\text {Abel }}, V_{\text {off }}\right)$ on a smaller and coarser lattice of $16^{3} \times 32$ at $\beta=6.0$, i.e., $a \simeq 0.105(14) \mathrm{fm}$.

\begin{tabular}{ccccc}
\hline & $\sigma\left[a^{-2}\right]$ & $A$ & $C\left[a^{-1}\right]$ & $\chi^{2} / N_{\mathrm{df}}$ \\
\hline$V$ & $0.0499(9)$ & $0.275(4)$ & $0.637(5)$ & 1.26 \\
$V_{\text {Abel }}$ & $0.0443(5)$ & $0.075(2)$ & $0.196(2)$ & 1.38 \\
$V_{\text {off }}$ & $-0.0019(3)$ & $0.166(2)$ & $0.427(2)$ & 3.51 \\
$V-V_{\text {Abel }}$ & $0.0058(10)$ & $0.200(4)$ & $0.440(5)$ & 1.05 \\
\hline
\end{tabular}

\section{Perfect Abelian dominance of quark confinement}

As a remarkable fact, we find almost perfect Abelian dominance [30] of the string tension, $\sigma_{\text {Abel }} \simeq \sigma$, on the fine and large-volume lattice of $32^{4}$ at $\beta=6.4$, as shown in Fig.2(a) and in Table 1. On the other hand, as shown in Table 2, we find only approximate Abelian dominance, $\sigma_{\text {Abel }} \simeq 0.9 \sigma$, on the smaller and coarser lattice of $16^{3} \times 32$ at $\beta=6.0$, which is consistent with the previous studies [25, 26].

To demonstrate the perfect Abelian dominance clearly, we perform the two fit analyses for $V(r)-V_{\text {Abel }}(r)$ on the $32^{4}$ lattice at $\beta=6.4$ in terms of i) Coulomb-plus-linear Ansatz of Eq. (17) and ii) pure Coulomb Ansatz of Eq. (17) with $\sigma=0$, respectively. As shown in Table 3, the former fit indicates no difference between the string tensions in $V(r)$ and $V_{\text {Abel }}(r)$ with almost perfect precision, and the latter fit indicates that $V(r)-V_{\text {Abel }}(r)$ is well described by a pure Coulomb Ansatz [30]. [See also the main panel of Fig.2(c).]

Thus, we conclude that the perfect Abelian dominance of the string tension, i.e., $\sigma_{\mathrm{Abel}} \simeq \sigma$, is observed on the fine and large-volume lattice of $32^{4}$ at $\beta=6.4$.

TABLE 3. Fit analysis for $V(r)-V_{\text {Abel }}(r)$ on the $32^{4}$ lattice at $\beta=6.4$. We list the best-fit parameter set $(\sigma, A, C)$ in the Coulomb-plus-linear Ansatz, and the best-fit parameter set $(A, C)$ in the Coulomb Ansatz with $\sigma=0$. These fit results indicate that $V-V_{\text {Abel }}$ takes a pure Coulomb form and has zero string tension.

\begin{tabular}{ccccc}
\hline & $\sigma\left[a^{-2}\right]$ & $A$ & $C\left[a^{-1}\right]$ & $\chi^{2} / N_{\mathrm{df}}$ \\
\hline$V-V_{\text {Abel }}$ & $-0.00017(22)$ & $0.223(3)$ & $0.433(2)$ & 0.86 \\
$V-V_{\text {Abel }}$ & - & $0.221(2)$ & $0.432(1)$ & 0.84 \\
\hline
\end{tabular}




\section{Simple nontrivial summation formula}

In contrast to Abelian dominance for the long-distance confinement properties, there is a significant difference between $V(r)$ and $V_{\mathrm{Abel}}(r)$ at short distances. For the accurate analysis of the short-distance behavior of the potential, the fine lattice at $\beta=6.4$ is preferable.

As shown in Fig.2(b), we newly find a simple but nontrivial summation formula [30] of

$$
V(r) \simeq V_{\mathrm{Abel}}(r)+V_{\mathrm{off}}(r)
$$

among the QQ $\bar{Q}$ potential $V(r)$, the Abelian part $V_{\text {Abel }}(r)$, and the off-diagonal part $V_{\text {off }}(r)$.

This summation formula (18) indicates that the significant difference between $V(r)$ and $V_{\text {Abel }}(r)$ at short distances is almost complemented by the off-diagonal part $V_{\text {off }}(r)$. In the non-Abelian theory, however, this simple summation formula (18) is fairly nontrivial, because the link-variables are not commutable, e.g.,

$$
\prod_{C} M_{\mu}(s) u_{\mu}(s) \neq \prod_{C} M_{\mu}(s) \prod_{C} u_{\mu}(s)
$$

and this non-Abelian nature generally leads to $W_{C}\left[U_{\mu}(s)\right] \neq W_{C}\left[M_{\mu}(s)\right] \cdot W_{C}\left[u_{\mu}(s)\right]$. Nevertheless, the summation formula (18) is observed especially on the fine and large-volume lattice of $32^{4}$ at $\beta=6.4$.

\section{SUMMARY AND CONCLUDING REMARKS}

We have studied MA projection of quark confinement in SU(3) QCD on a fine and large-volume lattice of $32^{4}$ at $\beta=6.4$. We have investigated the static QQ potential $V(r)$, its Abelian part $V_{\text {Abel }}(r)$, and its off-diagonal part $V_{\text {off }}(r)$, from the on-axis lattice data. Remarkably, we have found almost perfect Abelian dominance of quark confining force, i.e., $\sigma_{\mathrm{Abel}} \simeq \sigma$ for the string tension, on the fine and large-volume lattice. In addition, we have newly found a simple nontrivial summation relation of $V(r) \simeq V_{\text {Abel }}(r)+V_{\text {off }}(r)$.

Thus, in spite of the non-Abelian nature of QCD, quark confinement is entirely kept in the Abelian sector of QCD in the MA gauge. In other words, Abelianization of QCD can be realized without loss of quark confining force by MA projection, which can partially reduce the difficulty stemming from non-Abelian nature of QCD. In any case, such infrared Abelianization scheme of QCD would be meaningful to understand the quark confinement mechanism in the non-Abelian gauge theory of QCD.

\section{ACKNOWLEDGMENTS}

H.S. thanks Prof. V. G. Bornyakov for his valuable suggestions. H.S. is supported by the Grant for Scientific Research [(C) No.23540306] from the Ministry of Education, Science and Technology of Japan. N.S. is supported by a Grant-inAid for JSPS Fellows (Grant No. 250588) and by RIKEN iTHES Project. The lattice QCD calculations were partially performed on NEC-SX8R at Osaka University.

\section{REFERENCES}

1. Y. Nambu, in Preludes in Theoretical Physics, in honor of V. F. Weisskopf (North-Holland, Amsterdam, 1966).

2. M. Y. Han and Y. Nambu, Phys. Rev. 139, B1006 (1965)

3. D. J. Gross and F. Wilczek, Phys. Rev. Lett. 30, 1343 (1973); H. D. Politzer, Phys. Rev. Lett. 30, 1346 (1973).

4. Y. Nambu, Phys. Rev. D10, 4262 (1974); G. 't Hooft, in High Energy Physics, (Editorice Compositori, Bologna, 1975); S. Mandelstam, Phys. Rept. 23, 245 (1976).

5. Y. Nambu and G. Jona-Lasinio, Phys. Rev. 122, 345 (1961); Phys. Rev. 124, 246 (1961).

6. M. Creutz, Phys. Rev. Lett. 43, 553 (1979); Phys. Rev. D21, 2308 (1980).

7. H. J. Rothe, Lattice Gauge Theories, 4th ed. (World Scientific, 2012), and its references.

8. Y. Nambu, in Symmetries and Quark Models (Wayne State Univ., 1969); Lecture Notes at the Copenhagen Symposium (1970).

9. H. Ichie and H. Suganuma, Nucl. Phys. B574, 70 (2000); Nucl. Phys. B548, 365 (1999); Phys. Rev. D60, 077501 (1999).

10. G. 't Hooft, Nucl. Phys. B190, 455 (1981).

11. H. Suganuma, S. Sasaki, and H. Toki, Nucl. Phys. B435, 207 (1995);

H. Suganuma, S. Sasaki, H. Toki, and H. Ichie, Prog. Theor. Phys. Suppl. 120, 57 (1995). 
12. Z. F. Ezawa and A. Iwazaki, Phys. Rev. D25, 2681 (1982); Phys. Rev. D26, 632 (1982).

13. T. Suzuki, Prog. Theor. Phys. 80, 929 (1988); S. Maedan and T. Suzuki, Prog. Theor. Phys. 81, 229 (1989).

14. K.-I. Kondo, Phys. Rev. D57, 7467 (1998); K.-I. Kondo, S. Kato, A. Shibata, and T. Shinohara, Phys. Rept. 579, 1 (2015).

15. A. S. Kronfeld, G. Schierholz, and U.-J. Wiese, Nucl. Phys. B293, 461 (1987);

A. S. Kronfeld, M. L. Laursen, G. Schierholz, and U.-J. Wiese, Phys. Lett. B198, 516 (1987).

16. K. Amemiya and H. Suganuma, Phys. Rev. D60, 114509 (1999).

17. S. Gongyo, T. Iritani, and H. Suganuma, Phys. Rev. D86, 094018 (2012);

S. Gongyo and H. Suganuma, Phys. Rev. D87, 074506 (2013).

18. T. Suzuki and I. Yotsuyanagi, Phys. Rev. D42, 4257(R) (1990).

19. G. S. Bali, V. Bornyakov, M. Müller-Preussker, and K. Schilling, Phys. Rev. D54, 2863 (1996).

20. For a short review of Abelian dominance of quark confinement, M. I. Polikarpov, Nucl. Phys. Proc. Suppl. 53, 134 (1997).

21. J. D. Stack, S. D. Neiman, and R. J. Wensley, Phys. Rev. D50, 3399 (1994)

22. O. Miyamura, Phys. Lett. B353, 91 (1995); R. M. Woloshyn, Phys. Rev. D51, 6411 (1995).

23. H. Suganuma, A. Tanaka, S. Sasaki and O. Miyamura, Nucl. Phys. Proc. Suppl. 47, 302 (1996).

24. H. Suganuma, K. Amemiya, A. Tanaka, and H. Ichie, Nucl. Phys. A670, 40 (2000);

H. Suganuma, H. Ichie, A. Tanaka, and K. Amemiya, Prog. Theor. Phys. Suppl. 131, 559 (1998).

25. J. D. Stack, W.W. Tucker, and R.J. Wensley, Nucl. Phys. B639, 203 (2002).

26. V. G. Bornyakov, H. Ichie, Y. Koma, Y. Mori, Y. Nakamura, D. Pleiter, M. I. Polikarpov, G. Schierholz, T. Streuer, H. Stüben, and T. Suzuki (DIK collaboration), Phys. Rev. D70, 074511 (2004)

27. K. Langfeld, Phys. Rev. D69, 014503 (2004).

28. G. S. Bali and K. Schilling, Phys. Rev. D47, 661 (1993).

29. T. T. Takahashi, H. Matsufuru, Y. Nemoto, and H. Suganuma, Phys. Rev. Lett. 86, 18 (2001);

T. T. Takahashi, H. Suganuma, Y. Nemoto, and H. Matsufuru, Phys. Rev. D65, 114509 (2002).

30. N. Sakumichi and H. Suganuma, Phys. Rev. D90, 111501(R) (2014); Phys. Rev. D92, 034511 (2015). 